\title{
Alternativa mínimamente invasiva a la cirugía en sangrado renal espontáneo
}

\author{
Minimally invasive alternative to surgery in spontaneous renal \\ haemorrhage
}

\begin{abstract}
Paul Gamboa, Carlos M Fava, León Valdivieso, Gustavo Lev, Gaspar Caponi, Oscar A. Mendiz
\section{RESUMEN}

El sangrado renal espontáneo es una condición clínica de causas variadas siendo la principal la tumoral y en algunos casos no se logra determinar la etiología, el tratamiento debe ser conservador dependiendo de la situación clínica del paciente y en caso de compromiso hemodinámico se requiere nefrectomía de urgencia, que se asocia a una alta mortalidad. En los últimos años el manejo percutáneo se ha convertido en una opción alterna a la cirugía en pacientes con inestabilidad hemodinámica. En este escrito hemos querido reportar un caso de sangrado renal espontáneo mane-

\section{ABSTRACT}

Spontaneous renal bleeding is a clinical condition of varied causes, the main etiology is the tumour but, in some cases, etiology can't be defined. Treatment should be conservative depending on the clinical situation of the patient and in case of hemodynamic instability, emergency nephrectomy will be required associated with high mortality. In the last few years, percutaneous management has become an alternative option to surgery in patients with hemodynamic instability. In this paper we report a case of spontaneous renal bleeding successfully percutaneously managed.
\end{abstract} jado exitosamente por vía percutánea.

Palabras claves: sangrado renal espontáneo, embolización terapéutica, síndrome de Wünderlich.
Key words: bleeding, embolisation, Wünderlich syndrome

Revista Argentina de Cardioangiología Intervencionista 2019;10(2):83-85. https://doi.org/10.30567/RACI/201902/0083-0085

\section{INTRODUCCIÓN}

El sangrado renal espontáneo se define como la extravasación de sangre al espacio subcapsular y perirrenal sin que exista traumatismo externo, manipulación endourológica o endovascular previa; tiene diferentes etiologías y la neoplasia es la más frecuente ${ }^{1}$. El sangrado renal espontáneo es una patología poco frecuente que en la literatura se encuentra como reportes de casos y en ocasiones pequeñas series. Por tratarse de una entidad poco sospechada su diagnóstico puede ser dificultoso, lo que favorece un aumento en la morbilidad y mortalidad $^{2}$.

\section{OBJETIVO}

Reportar un caso de sangrado renal espontáneo manejado por vía percutánea de forma exitosa.

\section{DESCRIPCIÓN DEL CASO}

Se trata de un paciente masculino de 77 años con antecedente de reemplazo valvular aórtico con prótesis mecánica y cirugía de revascularización miocárdica, fibrilación auricular permanente, anticoagulación oral con warfarina y urolitiasis.

Consulta al departamento de emergencias por cuadro clíni-

1. ICyCC Instituto de Cardiología y Cirugía Cardiovascular. Hospital Universitario Fundación Favaloro. Departamento de Intervenciones por Cateterismo

$\triangle$ Correspondencia: Dr. Oscar Mendiz. ICyCC-Fundación Favaloro. Avda. Belgrano 1746. 40 Piso, (1093) CABA, Rep. Argentina.omendiz@ffavaloro.org

Los autores no declaran conflictos de intereses

Recibido: 22/03/2019| Aceptado: 29/05/2019 co de 24 horas de evolución de dolor lumbar izquierdo irradiado a hipogastrio y testículo ipsilateral. Niega otro síntoma asociado.

Laboratorio de ingreso: hematocrito $37 \%$; leucocitos 11,0 $\mathrm{mil} / \mathrm{mm}^{3}$; plaquetas $209 \mathrm{mil} / \mathrm{mm}^{3}$; uremia $60 \mathrm{mg} / \mathrm{dl}$; creatinemia $2,3 \mathrm{mg} / \mathrm{dl}$; sodio $136 \mathrm{mEq} / \mathrm{l}$; potasio $3,9 \mathrm{mEq} / \mathrm{l}$.

Tiempo de tromboplastina parcial (TTP) $55 \mathrm{~s}$ Tiempo de protrombina (TP) 80 s RIN 8,95.

Tomografía abdominal con contraste: riñón izquierdo con evidencia de imagen hiperdensa en semiluna subcapsular y periférica en probable relación con hematoma asociada a colección hemática que compromete los espacios perirrenales, pararrenal anterior ipsilateral y contralateral. Colección hemática que se extiende en mayor medida por encima del músculo psoas izquierdo (Figura 1).

A pesar de la corrección de los tiempos de coagulación el paciente evoluciona con caída abrupta del hematocrito a $26 \%$, hemoglobina $8,4 \mathrm{~g} / \mathrm{dl}$ e inestabilidad hemodinámica requiriendo transfusión de dos unidades de glóbulos rojos, reanimación hídrica y soporte vasopresor. Se interpreta cuadro clínico como un sangrado renal espontáneo con shock hipovolémico. Por alto riesgo de nefrectomía se decide manejo percutáneo.

\section{TÉCNICA}

Bajo sedación consciente se realizó una punción radial izquierda con un set introductor 5 French (Fr). Con un catéter Judkins de derecha 3.5 montado sobre un alambre guía hidrofílico 0,35 " se realiza una arteriografía renal izquierda evidenciando extravasación de contraste a nivel de dos arterias arqueadas dependientes de arteria segmentaria inferior (Figura 2).

A través de microcatéter (Progreat, Terumo corporation, Ashitaka, Japón) se realiza embolización selectiva con espon- 


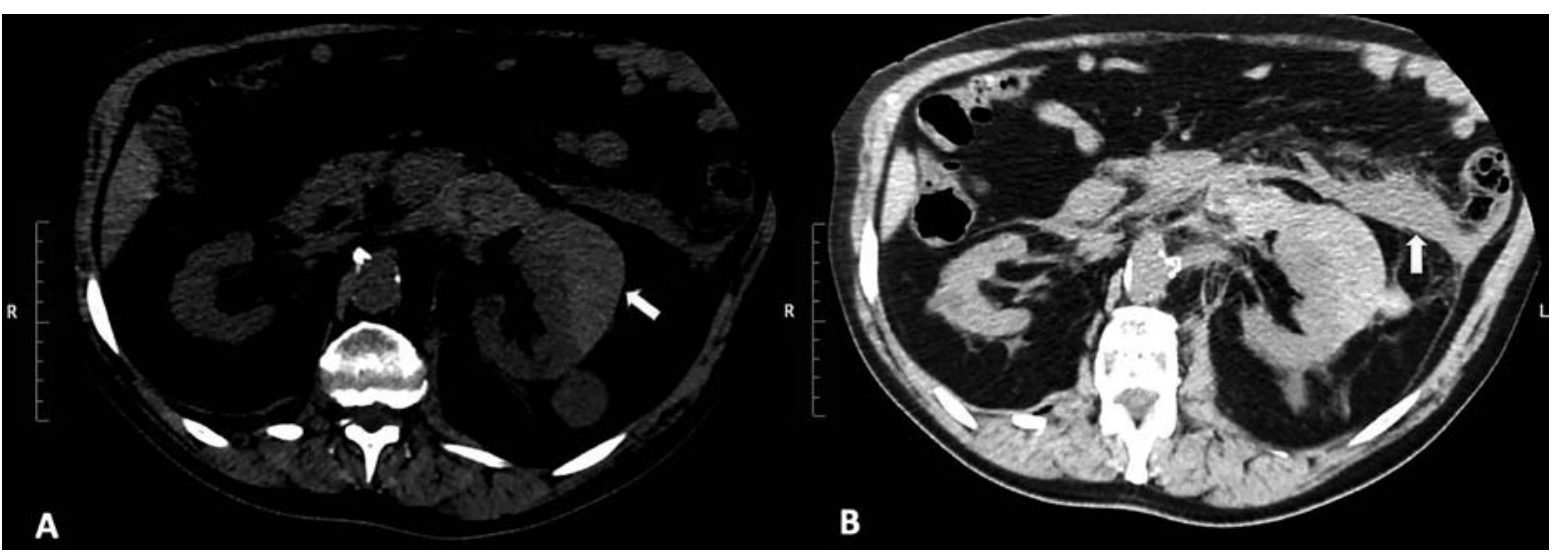

Figura 1. Tomografía de abdomen simple. A. Riñón izquierdo con evidencia de imagen hiperdensa en semiluna subcapsular (flecha). B. Colección hemática que se extiende en mayor medida por encima del músculo psoas izquierdo (flecha).

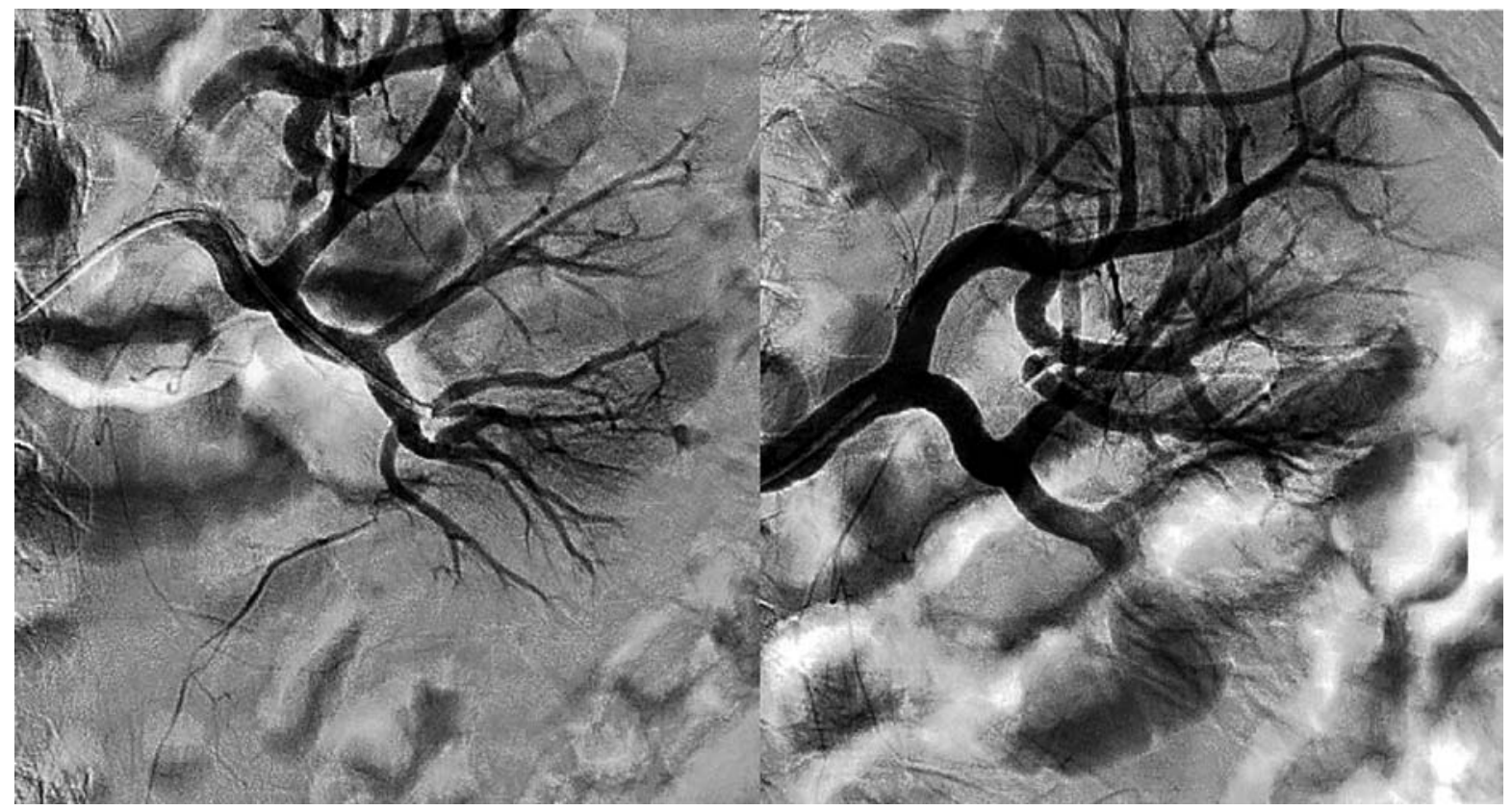

Figura 2. Extravasación de contraste a nivel de dos arterias arqueadas dependientes de arteria segmentaria inferior.

ja de gelatina (Spongostan, Johnson \& Johnson Institute, California, USA) logrando control del sangrado (Figura 3). Cuarenta y ocho horas posterior al procedimiento, el paciente presenta mejoría de dolor lumbar, estabilidad hemodinámica, niveles de hematocrito constantes y mejoría de azoados por lo cual es dado de alta. En el control a los 30 días el paciente continúa asintomático con normalización de la función renal y reinicio de la anticoagulación oral.

\section{DISCUSIÓN}

El sangrado renal espontáneo, también conocido como síndrome de Wünderlich, es una condición clínica descrita por primera vez en 1700 por Bonet ${ }^{3}$. Sus causas son variadas, la principal es la tumoral y en algunos casos no se logra determinar la etiología. Su inicio puede ser súbito o insidioso y su diagnóstico es dificultoso, dependiendo mucho de la clínica y la tomografía de abdomen ${ }^{4}$. El tratamiento debe ser conservador dependiendo de la situación clínica del paciente y en caso de compromiso hemodinámico se requiere nefrectomía de urgencia que se asocia a una alta morbilidad y mortalidad 5 . La evolución de los materiales empleados y el perfeccionamiento de la técnica para la embolización lo han convertido en un procedimiento seguro con pocas complicaciones y mínimo impacto en la función renal cuando se hace de forma selectiva, lo que lo convierte en una técnica alternativa a la cirugía evitando en muchos casos la nefrectomía ${ }^{6,7}$. En nuestro caso se consiguió controlar el sangrado renal sin compromiso de la función renal a través de técnica percutánea. La mayoría de las embolizaciones renales se pueden realizar con anestesia local, pero cuando se realiza con etanol o agentes embólicos no adhesivos el dolor que estos producen pueden justificar el uso de anestesia general ${ }^{8}$. En cuanto al agente embolizante no existe un consenso en su elección. La esponja de gelatina (Spongostan, Johnson \& Johnson institute, California, USA) es de uso muy frecuente y al ser de un material biodegradable induce una oclusión temporal de 3 a 90 días. A diferencia de los otros agentes de oclusión proximal permanentes como los coils metálicos, de los que existen diferentes combinaciones de formas y tamaño; sin embargo, son materiales costosos y deberían ser reservados para embolizaciones dificultosas, así como los tapones vasculares (vascular plugs) (Amplatzer, St. Jude Medical Inc., USA), los cuales 


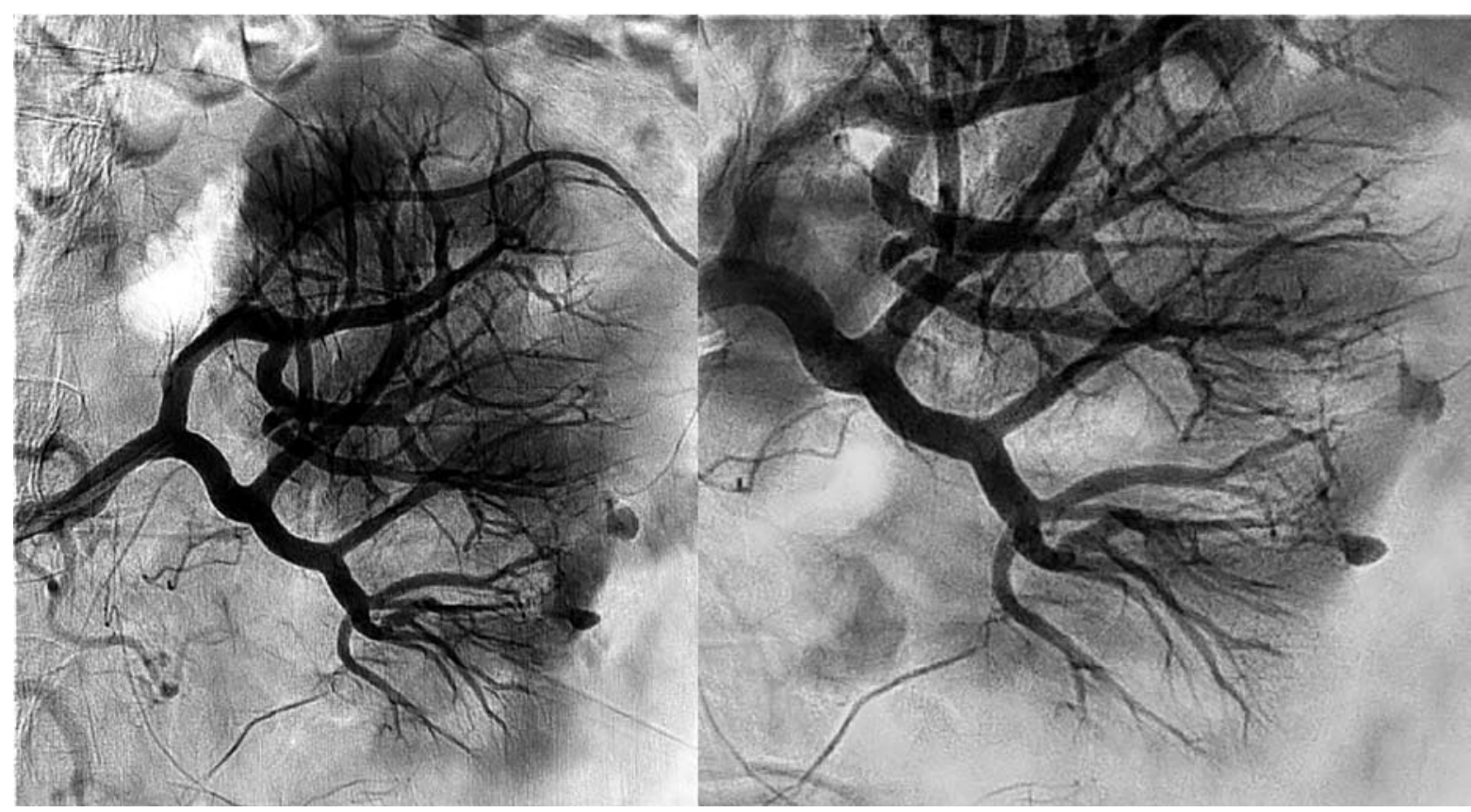

Figura 3. A. Embolización selectiva con esponja de gelatina. B. Arteriografía final logrando control del sangrado.

proveen una rápida embolización y son ideales para situaciones de arterias de gran tamaño y alto flujo?. En nuestro caso al tratarse de una arteria segmentaria de pequeño tamaño y no evidenciarse malformaciones arteriovenosas se decidió por una oclusión temporal con esponja de gelatina.

\section{CONCLUSIÓN}

La embolización de la arteria renal mediante técnica percutánea es una alternativa segura para el manejo del sangrado renal espontáneo.

\section{BIBLIOGRAFÍA}

1. 1. Polkey HJ, Vynalek WJ. Spontaneous nontraumatic perirenal and renal hematomas. An experimental and clinical study. Arch Surg 1933;26:196

2. 2. Ivascu FA, Janczyk RJ, Bair HA. Spontaneous retroperitoneal hemorrhage. Am J Surg 2005; 189(3):345-7.

3. 3. Wünderlich CRA. Handbuch der pathologie und therapie. Ebner \& Seubert 1976; Stuttgart.

4. 4. Sebastia MC, Perez-Molina MO, Alvarez-Castells A. CT evaluation of underlying cause in spontaneous sub-capsular and perirenal hemorrhage. Eur Radiol 1997;7:686-90

5. 5. Kendall AR, SenayBA. Spontaneoussubcapsularrenal hematoma:diagnosis and management. J Urol 1988;139:246.

6. 6. Jacobson Al, Amukele SA, Marcovich R. Efficacy and morbidity of therapeutic renal embolization in the spectrum of urologic disease. J Endourol 2003;17:385-91.

7. 7. Pappas P. Leonardou P. Papadoukakis S. Urgent superselective segmental renal artery embolization in the treatment of life-threatening renal hemorrhage. Urol Int 2006;77:34-41.

8. 8. Ginat DT, Saad WE, Turba UC. Transcatheter renal artery embolization: clinical applications and techniques. Tech Vasc Interv Radiol 2009;12:224-39.

9. 9. Loffroy R, Rao P, KwakBK. Transcatheter arterial embolization in patients with kidney diseases: an overview of the technical aspects and clinical indications. Korean J Radiol 2010;11:257-68 